\title{
ESTUDO COMPARATIVO DE MÉTODOS DE AMOSTRAGEM DE COMUNIDADES DE COSTÃO
}

\author{
SABINO, C. M. ${ }^{1}$ e VILLAÇA, R. ${ }^{2}$ \\ ${ }^{1}$ Mestranda da Universidade Federal Fluminense, Programa de Pós-graduação em Biologia Marinha \\ ${ }^{2}$ Universidade Federal Fluminense, Programa de Pós-graduação em Biologia Marinha, C.P. 100436, \\ CEP 24001-970, Niterói, RJ, Brasil \\ Correspondência para: Roberto Villaça, Universidade Federal Fluminense, Programa de Pós-graduação \\ em Biologia Marinha, C.P. 100436, CEP 24001-970, Niterói, RJ, Brasil, e-mail: gbmbeto@vm.uff.br \\ Recebido em 22/04/98 - Aceito em 11/09/98 - Distribuído em 10/09/99
}

(Com 8 figuras)

\begin{abstract}
Sampling methods for rocky shores

This study compares three sampling methodologies of rocky shore communities: visual estimation, point intercept quadrats and destructive sampling. Visual estimation was performed in two sizes of sampling device and, point intercept quadrats was done with different numbers of marked dots. The sampling took place on the rocky shore of Forno beach, Búzios, RJ, in two different algae belts; one whose the landscape is characterized by Amphiroa sp., the other, Sargassum furcatum was the most conspicuous species. The data were analyzed by ANOVA and Kruskal-Wallis tests. Number of species, total percent cover, diversity and evenness indices were the variables used. The results shown that in general the methods compared were not statistically different one from the other in this community. There were significative differences between the methods when comparison was made between nondestructive and destructive sampling. However, smaller areas of sampling devices and fewer intercept points decreased the sampling power in respect of sampling species richness. All of the methods studied were considered similar in concern of total cover index and of diversity and evenness indices, with some exceptions. Present data are useful only for rocky communities with similar profiles. The choice of method must be made in regard of each situation.
\end{abstract}

Key words: sampling methods, rocky shore, phytobenthos, Búzios, RJ.

\section{RESUMO}

O presente estudo compara três metodologias de amostragem de costão rochoso: estimativa visual, pontos de interseção e raspagem total do substrato. No método de estimativa visual, variou-se o tamanho do amostrador e, no de pontos de interseção, o número de pontos marcados. As amostras foram coletadas no costão da praia do Forno, Búzios, RJ, em duas faixas, uma dominada fisionomicamente por Amphiroa sp. e a outra, por Sargassum furcatum. Foram aplicados testes de ANOVA e KruskalWallis aos resultados encontrados, utilizando-se como variáveis os índices bionômicos de número de espécies, porcentagem total de recobrimento, índice de diversidade e índice de uniformidade. Os resultados provaram que nenhuma metodologia de amostragem semelhante mostrou-se estatisticamente diferente da outra. Existe diferença quando se comparam os métodos não-destrutivos ao método destrutivo. No entanto, amostradores com menor área ou menor número de pontos não amostram bem o índice número de espécies. Os métodos não apresentaram diferença significativa em relação à cobertura total e aos índices de diversidade e uniformidade, salvo algumas exceções. Os resultados do presente trabalho são aplicáveis apenas a comunidades de costão rochosos com perfis similares ao da comunidade estudada. A escolha do método deve ser considerada para cada situação.

Palavras-chave: metodologia de amostragem, costão rochoso, fitobentos, Búzios, RJ. 


\section{INTRODUÇÃO}

No estudo de estrutura de comunidades é essencial a adequação dos métodos de amostragem aos objetivos específicos do trabalho a ser desenvolvido. Desde a escolha do local de estudo até o desenho experimental, todas são fases de um procedimento de amostragem que deve visar coletar dados o mais adequadamente possível, tendo em mente as hipóteses que se quer testar.

A maior parte dos estudos, aparentemente, procede mais pelo costume e tradição, seguindo metodologias já consagradas e descritas, do que pela consideração cuidadosa de padrões potenciais e dos problemas inerentes à amostragem de diferentes organismos em diferentes regiões (GreigSmith, 1983; Andrew \& Mapstone, 1987; Kingston \& Riddle, 1989).

$\mathrm{Na}$ tentativa de procurar entender os padrões de distribuição e abundância das comunidades assume-se, implicitamente, que os métodos empregados fornecem uma estimativa precisa $\mathrm{e}$ consistente do número de organismos realmente presentes (Meese \& Tomich, 1992). Mas, é preciso considerar que as informações sobre a distribuição e abundância dos organismos são geralmente a única base para decisões ecológicas e de manejo. A descrição precisa e exata de um padrão é, portanto, essencial para a maioria dos aspectos ecológicos (Andrew \& Mapstone, 1987).

É absolutamente impossível amostrar toda uma comunidade de um dado ecossistema, por isso, o tipo de amostragem escolhido deve ser considerado o mais representativo da comunidade em questão. O problema da representatividade é básico em qualquer estudo ecológico - uma boa amostra deve oferecer uma imagem, se possível, completa, qualitativa e quantitativamente da comunidade estudada (Rosso, 1995). A partir daí, surgem as questões relacionadas ao dimensionamento amostral, como a eficiência do método de amostragem e o número de amostras, procurando sempre respeitar a relação exatidão/precisão.

Implícito no tamanho do amostrador está a questão da área amostral mínima. Boudouresque (1974) definiu-a como a menor área onde ainda se observa a estrutura geral da comunidade e, por conseguinte, onde melhor estão representadas as espécies que ocorrem nessa comunidade. Assim, as áreas mínimas tendem a aumentar em comunidades de maior complexidade estrutural. Essa é uma questão bastante discutida e ainda sem resultados conclusivos. Sinteticamente, o tamanho do amostrador deve ser compatível com as características da comunidade e o arranjo espacial que se queira estudar ou revelar (Andrew \& Mapstone, 1987; Rosso, 1995).

A preocupação com a forma do amostrador é particularmente interessante em situações em que ela possa se adequar à forma de agregação dos organismos ou às feições topográficas que influenciem na disposição dos organismos (Andrew \& Mapstone, 1987). No entanto, pouco é feito no sentido de melhor esclarecer como a forma interfere nos resultados. A adoção quase generalizada de elementos quadrados (ou retangulares) é todavia indiscutível (Rosso, 1995).

Apesar da importância do tamanho e da forma do amostrador, a parte mais crítica de um planejamento amostral é a determinação do número de amostras. É esse índice que vai conferir maior precisão à metodologia aplicada. A precisão é o grau de concordância entre um número de medidas, ou estimativas, para uma mesma população. A precisão reflete-se na variabilidade de uma estimativa e é um atributo do procedimento amostral e, não, um reflexo de alguma característica da população que está sendo amostrada (Andrew \& Mapstone, 1987). Ainda segundo esses autores, a utilidade de uma estimativa é dependente da sua precisão, assim como da exatidão. Exatidão é a proximidade de uma medida, ou estimativa, do valor de uma variável medida ou do parâmetro que está sendo estudado.

Assim, apesar de os métodos disponíveis para amostragem serem muitos e variados, os dados coletados têm duas implicações comuns a todos eles: 1. todos estão sujeitos a problemas de inexatidão e imprecisão provenientes da aplicação dos métodos amostrais; 2 . todos os profissionais são limitados por tempo e recursos financeiros, o que restringe a localização e o número de amostras que podem ser feitas (Andrew \& Mapstone, 1987).

Existem diferentes métodos de amostragem de bentos de substrato duro. Acredita-se que cada um tenha aplicações específicas, características de exatidão, precisão, repetibilidade e consistência também diferentes. A escolha da metodologia mais adequada associa o conhecimento teórico do método ao tipo de ambiente a ser estudado. Portanto, é necessário o conhecimento prévio das caracte- 
rísticas de uma determinada comunidade, bem como dos fatores ambientais que podem influenciar na sua ocorrência. A densidade populacional, o tamanho e o modo de dispersão dos indivíduos exigem uma dimensão amostral própria. Um método próprio para uma comunidade pode não ser para outra.

A comparação entre os métodos, de forma a identificar o mais representativo da realidade da comunidade e o mais adequado a ser utilizado, tem instigado alguns estudos recentes (Meese \& Tomich, 1992; Dethier et al., 1993), apesar de a metodologia da amostragem em si já ser um objeto de discussão muito antigo.

Estudos comparativos de metodologias têm sido desenvolvidos para avaliar criticamente a eficácia relativa de cada método sob reais condições de campo. Meese \& Tomich (1992) examinaram cinco métodos de estimativa de recobrimento: estimativa visual, fotografia, pontos de interseção randômicos, pontos de interseção com distribuição regular e pontos de interseção estratificados randomicamente. Dethier et al. (1993) avaliaram os métodos de estimativa visual e pontos de interseção com amostras no campo e simulações no computador. Foster et al. (1991) compararam os métodos de pontos de interseção com o de fotografias dos quadrados amostrados. Todos os estudos utilizaram resultados de porcentagem de recobrimento para fins comparativos.

Embora sabendo-se das particularidades inerentes a cada ambiente e comunidade, estudos dessa natureza são valiosos à medida que fornecem a base sobre a qual decisões podem ser tomadas a cerca de qual método é mais apropriado a um determinado conjunto de circunstâncias ambientais (Meese \& Tomich, 1992). Embora isso não inutilize a importância de uma amostragem preliminar, muito pelo contrário, vem confirmar a necessidade de tal procedimento e adicionar informações sobre os vários métodos disponíveis. De certa forma, trabalhos dessa natureza fornecem maiores subsídios aos pesquisadores para um planejamento mais conciso desse importante estágio do dimensionamento amostral, que atualmente está tão subestimado.

O presente estudo tem como objetivo comparar três das mais utilizadas metodologias de amostragem de comunidades de costão rochoso: estimativa visual (John et al., 1977), pontos de interseção (Jones et al., 1980) e raspagem total do substrato (Boudouresque, 1971; Murray \& Littler, 1978; Yoneshigue, 1985; Villaça, 1988). No entanto, além de comparar os métodos através da porcentagem de recobrimento, também foram utilizados os parâmetros de descrição da comunidade: número de espécies, índice de diversidade e índice de uniformidade.

\section{ÁREA DE ESTUDO}

Este estudo foi desenvolvido no costão esquerdo da praia do Forno, no município de Búzios, litoral norte do Estado do Rio de Janeiro, local que se destaca como uma das regiões mais preservadas do Estado.

Geomorfologicamente é uma praia localizada no fundo de uma longa enseada voltada para sudoeste, formada por costões dos dois lados e uma pequena faixa de areia de aproximadamente 150 $\mathrm{m}$. Fica protegida de uma situação de mar aberto pela sua própria conformação e pela ponta da Lagoinha (Fig. 1).

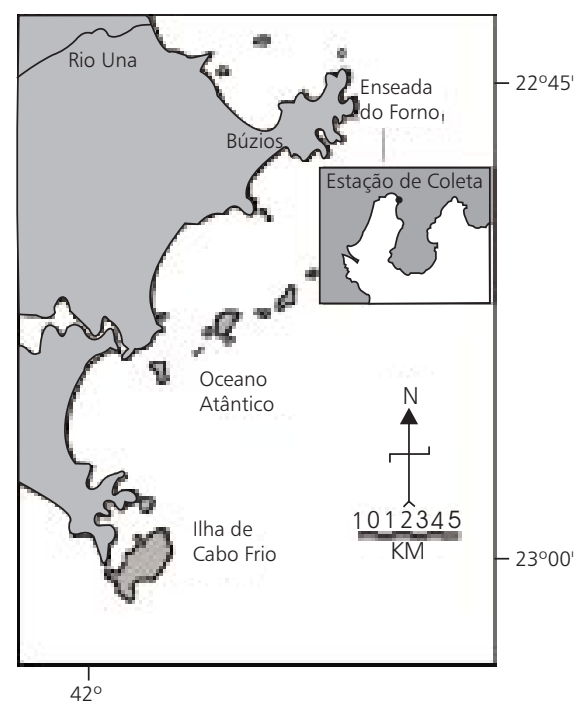

Fig. 1 - Localização geográfica da estação de coleta, praia do Forno, Búzios, RJ.

A área de costão escolhida para o estudo não tem uma inclinação muito acentuada, aproximadamente 20 a $30^{\circ}$, o que permite que durante o período de maré de baixamar de sizígia, uma faixa de aproximadamente $7 \mathrm{~m}$, fique emersa durante quase $2 \mathrm{~h}$. $\mathrm{O}$ modo é predominantemente calmo, podendo ficar batido com a entrada de ventos de 
SW ou SE. Nessa área, o costão é dominado fisionomicamente pelas algas Amphiroa spp., Jania adhaerens, Sargassum furcatum e pelo ouriço Echinometra lucunter.

\section{MATERIAL E MÉTODOS \\ Trabalho de campo}

Os métodos utilizados foram: estimativa visual (EV), estimativa por pontos de interseção (PI) (métodos não-destrutivos) e raspagem (RT) (método destrutivo). Para o método de estimativa visual foram utilizados dois tamanhos de quadrados.

Foram escolhidas duas faixas distintas da comunidade entre marés e franja do infralitoral: uma dominada fisionomicamente pela alga rodofícea calcária Amphiroa spp. e outra, em que a alga feofícea Sargassum furcatum é a mais conspícua. A extensão do costão estudado é de aproximadamente $5 \mathrm{~m}$.

Na faixa de Amphiroa, os dados de cobertura foram obtidos utilizando-se os três métodos citados acima, enquanto na faixa de $S$. furcatum foram empregados apenas os métodos de estimativa visual e o de pontos de interseção.

O método de estimativa visual foi utilizado conforme descrito por John et al. (1977). Foram utilizados quadrados de $400 \mathrm{~cm}^{2}$ (EV20) divididos em 25 subquadrados e quadrados de $100 \mathrm{~cm}^{2}$ (EV10), também subdivididos em 25 quadrados.

O recobrimento foi estimado atribuindo-se o valor 1 (um) a espécie que ultrapassasse, em recobrimento, metade da área do subquadrado. Caso duas espécies ocupassem igualmente o subquadrado, as duas recebiam o valor 1 (um). Quando a predominância de uma espécie não fosse observada em algum dos subquadrados, nenhum valor era anotado. Para o cálculo da porcentagem de recobrimento utilizou-se o somatório de vezes que a alga foi encontrada nos subquadrados, dividido pelo número total de subquadrados, multiplicando-se o resultado por 100 .

O método de pontos de interseção foi utilizado conforme descrito por Meese \& Tomich (1992). O procedimento para atribuição de valores às espécies encontradas foi o mesmo que o utilizado na estimativa visual. No presente estudo, optamos por testar quadrados de $400 \mathrm{~cm}^{2}$ com 10 (PI10), 30 (PI30) e 50 (PI50) pontos de interseção marcados em uma malha de 100 pontos. As in- terseções foram marcadas com o auxílio de uma tabela de números aleatórios. Já o método de raspagem total, consistiu na retirada de todos os organismos que estavam recobrindo a superfície rochosa. Optou-se por um quadrado de $400 \mathrm{~cm}^{2}$ para delimitar a área de raspagem. Esse método foi utilizado somente na faixa de Amphiroa.

Para cada método foram feitas 5 replicações, dentro da extensão de $5 \mathrm{~m}$. A replicação foi feita sorteando-se um valor na tabela de números aleatórios a cada $1 \mathrm{~m}$ de extensão do costão, definindo, assim, a distância mínima entre uma réplica e outra. No total foram realizadas 55 amostras, 30 na faixa de Amphiroa e 25 na faixa de Sargassum furcatum. As amostragens foram efetuadas entre Abril e Julho de 1995, preferencialmente nos períodos de baixamar de sizígia.

\section{Tratamento de dados}

Os índices bionômicos de estrutura de comunidade utilizados foram: $n$, número total de espécies presentes em cada réplica e em cada tratamento; $\mathrm{n}_{\mathrm{v}}$, número de espécies vegetais; $\mathrm{n}_{\mathrm{a}}$, número de espécies animais; $\mathrm{R}_{i}$, porcentagem da superfície do substrato coberta pela espécie " $i$ "; $R_{t}$, somatório dos $\mathrm{R}_{\mathrm{i}}$ de todas as espécies de uma réplica, podendo ser superior a 100 pela possibilidade de haver mais de um estrato de vegetação. Foram calculados também, H', índice de diversidade de Shannon; E, índice de uniformidade para cada réplica. Para o cálculo dos índices H' e E, utilizou-se as fórmulas apresentadas em Legendre \& Legendre (1983).

A diferença entre os tratamentos (métodos) foi testada através de Análise de Variância, utilizando-se como variáveis número de espécies e os índices de diversidade e uniformidade calculados para cada tratamento e respectivas réplicas. No caso da porcentagem total de recobrimento foi utilizado o teste Kruskal-Wallis. O método de raspagem total foi comparado aos demais métodos, considerando-se apenas os resultados obtidos na faixa de Amphiroa.

Quando detectada diferença significativa ( $p \leq$ $0,05)$ nos resultados das Análises de Variância, foi utilizado o teste de Duncan para comparação de médias a posteriori. Todos os testes estatísticos foram feitos no programa Statistica (StatSoft, Inc., 1993). 


\section{RESULTADOS}

A comparação entre os métodos utilizados foi realizada através dos resultados obtidos para os índices bionômicos: número de espécies, porcentagem de recobrimento, índice de diversidade e índice de uniformidade, conforme exposto a seguir. O resultado das Análises de Variância entre os tratamentos de estimativa visual (EV) e pontos de interseção (PI) em relação a cada índice bionômico está apresentado na Tabela 1, abaixo.

\section{TABELA 1}

Valores de $p$ resultantes dos testes de ANOVA aplicados aos experimentos com os métodos de estimativa visual e pontos de interseção em relação às variáveis: número de espécies, índice de diversidade e índice de uniformidade.

\begin{tabular}{|l}
\hline Variáveis \\
\hline Número de espécies \\
Índice de diversidade \\
Índice de uniformidade \\
\hline
\end{tabular}

Na Tabela 2 estão apresentados os resultados dos testes de Análise de Variância em que, além dos tratamentos de estimativa visual e pontos de interseção, foram também considerados os resultados obtidos através do método de raspagem total (RT).

\section{TABELA 2}

Valores de $\boldsymbol{p}$ resultantes dos testes de ANOVA aplicados aos experimentos com os métodos de estimativa visual, pontos de interseção e raspagem total em relação às variáveis: número de espécies, índice de diversidade e índice de uniformidade.

\begin{tabular}{|l|l|}
\hline Variáveis & \multicolumn{1}{c|}{$\boldsymbol{p}$} \\
\hline Número de espécies & $0,00000^{* *}$ \\
\cline { 2 - 2 } Índice de diversidade & $0,04303^{*}$ \\
\hline Índice de uniformidade & $0,00004^{* *}$ \\
\cline { 2 - 2 }
\end{tabular}

\section{Número de espécies}

A comparação dos métodos em relação ao número de espécies mostrou que há diferença significativa entre os métodos, $p<0,01$ (Tabela 1). O resultado do Teste de Duncan mostrou que os métodos de menor área (EV10) e menor número de pontos de interseção (PI10) equivalem-se, assim como os métodos de tamanho intermediário (EV20 e PI30) (Fig. 2). PI50 foi o único método considerado diferente dos demais. De fato, foi o mé- todo mais eficiente, registrando maior número de espécies, situação que se reflete na maior média entre os cinco tratamentos. No entanto, também apresentou maior variabilidade nos valores obtidos (Fig. 2).

EV10 e PI10 registraram os menores número de espécies, porém, apresentaram valores médios bem próximos ( 3,2 e 3,8, respectivamente); o mesmo observa-se para EV20 e PI30 $(5,1$ e 5,6), mas com valores mais próximos de PI50 (Fig. 2).

A comparação incluindo o método de raspagem total apresentou diferença significativa, $p<0,01$ (Tabela 2). O Teste de Duncan identificou como diferente o tratamento de raspagem total. De fato, esse método, por retirar toda a área amostrada, permite um exame mais detalhado das espécies presentes, registrando maior número de espécies.

A Fig. 3 permite uma boa visualização desse resultado. Nela é bem visível a diferença do método de raspagem total em relação aos demais, e evidencia a sobreposição dos demais métodos. EV10, PI10 e PI30 formaram um grupo de valores médios mais próximos mas não caracterizaram forte diferença em relação a EV20 e PI50. Observa-se também a ampla variação dos resultados obtidos a partir de RT.

\section{Porcentagem de recobrimento}

A comparação entre os métodos em relação à porcentagem de recobrimento foi testada através do teste de Kruskal-Wallis, que não detectou diferença entre os métodos $(p=0,0521)$, mostrando que, quando se considera isoladamente porcentagem de recobrimento, todos os métodos fornecem resultados semelhantes. Na Fig. 4 está representada a distribuição das médias. Observa-se que, de fato, os valores obtidos em cada método ficaram praticamente em uma mesma faixa de valores. EV10, EV20 e PI50 apresentaram as maiores variabilidades internas.

\section{Índice de diversidade}

Comparando-se os métodos através do índice de diversidade, o resultado do teste de ANOVA indicou diferenças significativas, $p<0,01$ (Tabela 1).

O resultado do Teste de Duncan separou apenas o tratamento de menor área, que pode ser a causa dos menores valores de diversidade obtidos (Fig. 5). 


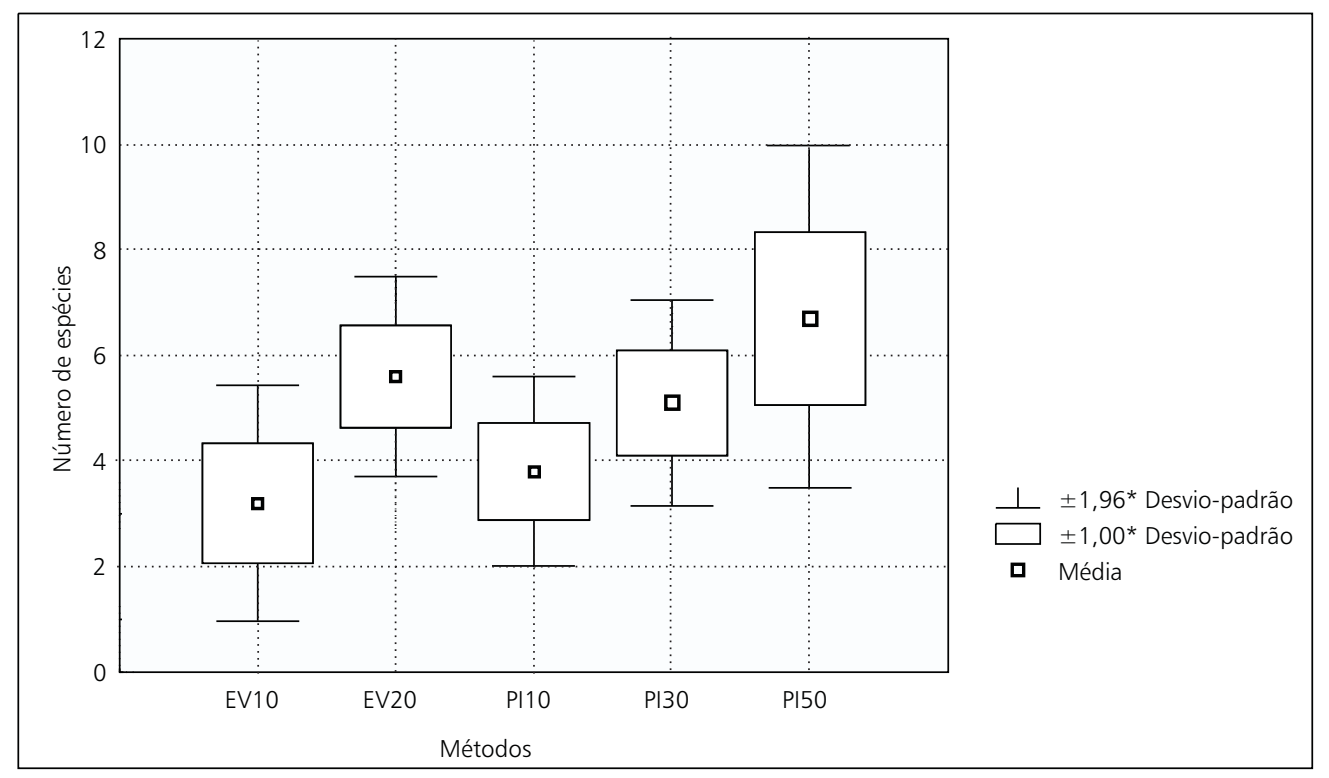

Fig. 2 - Número médio de espécies em cada método (EV10 e EV20, estimativa visual 10 x $10 \mathrm{~cm}$ e 20 x $20 \mathrm{~cm}$; PI10,30 e PI50, pontos de interseção com 10, 30 e 50 pontos).

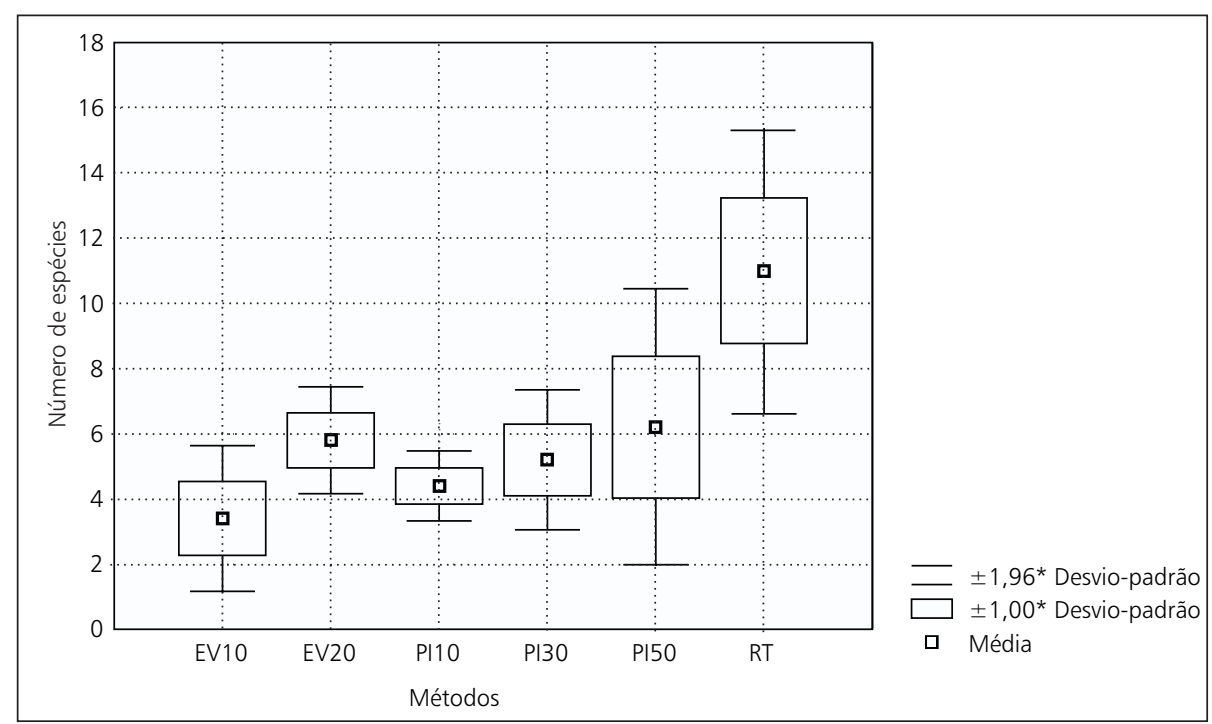

Fig. 3 - Número médio de espécies obtido no seis métodos (EV10 e EV20, estimativa visual 10 x $10 \mathrm{~cm}$ e 20 x $20 \mathrm{~cm}$; PI10, 30 e PI50, pontos de interseção com 10, 30 e 50 pontos; RT, raspagem total).

O tratamento PI10, o próximo na escala de médias, com o segundo menor valor de diversidade, foi agrupado com os demais, fato que pode estar relacionado com a área do quadrado e, não somente, com o número de pontos marcados. Embora, esse seja um número reduzido de pontos, a chance de registrar espécies diferentes aumenta com o tamanho da área delimitada pelo quadrado $-400 \mathrm{~cm}^{2}$.

Na Fig. 5, observa-se que os métodos que foram considerados semelhantes ficaram todos dentro de uma mesma faixa de valores médios e apresentaram variabilidades menores e mais homogêneas se comparadas àquelas apresentadas por 
EV10. Neste aspecto, o agrupamento encontrado parece estar relacionado preferencialmente com o tamanho dos quadrados, ou seja, quando considera-se a variável diversidade não importa a metodologia de amostragem se os amostradores têm as mesmas dimensões. Em relação à comparação com o método de raspagem total, o resul- tado do teste ANOVA, utilizando índice de diversidade, indicou diferença significativa, $\operatorname{com} p<$ 0,05 (Tabela 2).

O Teste de Duncan separou os métodos em dois grupos que se sobrepõem. Os métodos PI10 e PI30 aparecem nos dois grupos. O método EV10 é o único que fica isolado dos demais.

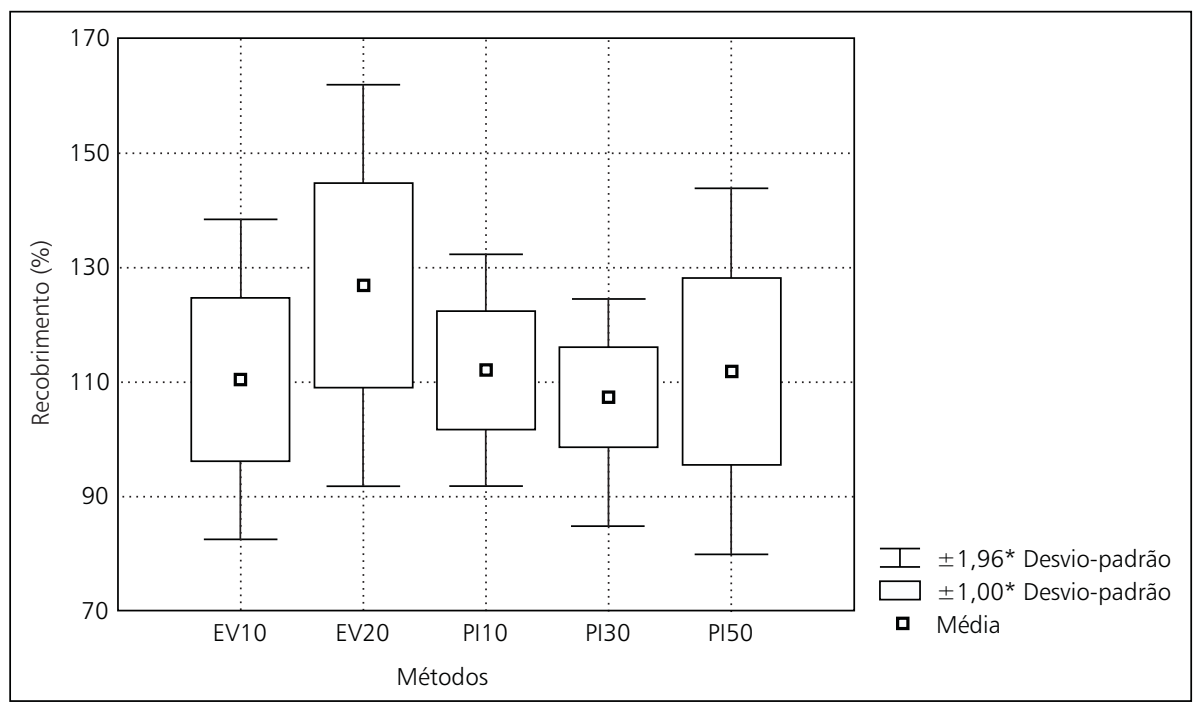

Fig. 4 - Porcentagem média de recobrimento em cada método (siglas conforme explicitado na Fig. 2).

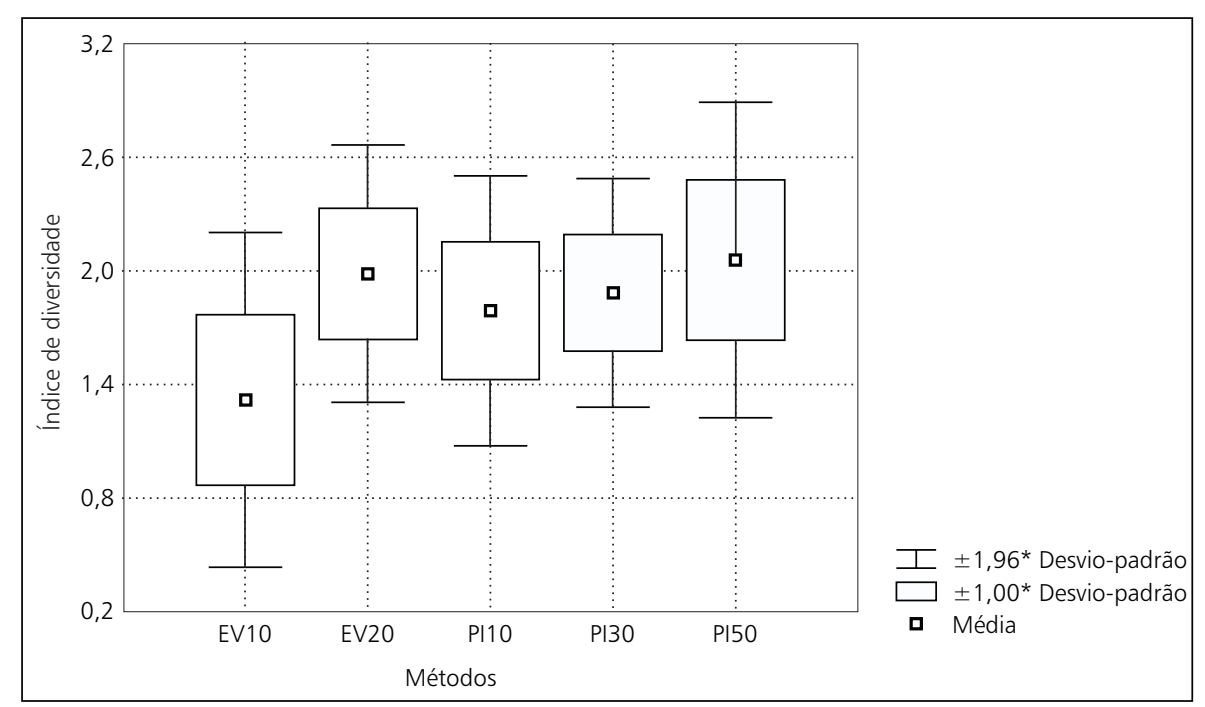

Fig. 5 - Médias dos índices de diversidade obtidas em cada método (siglas conforme explicitado na Fig. 2). 
Os métodos que compõem o primeiro grupo (EV10, PI30 e PI10) foram aqueles que apresentaram as menores médias (Fig. 6). O segundo grupo (PI30, PI10, EV20, PI50 e RT) apresenta valores médios mais próximos. Mas o fato de EV10 ser o único a ficar isolado - não houve superposição com o segundo grupo - é evidenciado pelo menor valor médio obtido e uma das maiores variabilidades. O maior valor médio de diversidade e a menor variabilidade de resultados foi do método RT $(2,26)$, não sendo, no entanto, considerada diferente dos demais, o que ocorreu apenas com EV10.

\section{Índice de uniformidade}

Em termos de índice de uniformidade, a comparação entre os métodos apresentou diferenças significativas, $p<0,01$ (Tabela 1). O resultado do Teste de Duncan mostrou que a diferença está no método PI10, que apresentou o maior valor médio de uniformidade $(0,95)$. Os demais métodos formaram um só grupo com valores médios que variaram de 0,77 a 0,83 .

Esse resultado evidencia que para o índice de uniformidade é mais relevante uma amostragem em que os métodos considerem toda a área amostrada ou o maior número de pontos de interseção possível. Uma grande área de quadrado, com poucos pontos de interseção marcados, possibilita uma amostragem tendenciosa a registrar apenas o organismo dominante, resultando em um índice que exagera na uniformidade da amostra.

Na Fig. 7, observa-se que, embora, as médias dos tratamentos de estimativa visual e pontos de interseção de 30 e 50 pontos tenham ficado muito próximas, a variabilidade dos resultados foi muito grande, com exceção do tratamento PI10 que apresentou a maior média $(0,95)$ e a menor variabilidade.

Incluindo-se o método de raspagem total no teste de comparação, utilizando-se o índice de uniformidade, foi detectada diferença significativa, com $p<0,01$ (Tabela 2). O Teste de Duncan indicou que raspagem total é diferente dos demais. A Fig. 8 evidencia esta diferença, mostrando que a média dos índices de uniformidade obtidos a partir da raspagem total foi a menor $(0,66)$ e está bastante fora do intervalo em que ficaram as demais. Os métodos RT e PI10 apresentaram as menores variabilidades.

\section{DISCUSSÃO}

Os índices bionômicos utilizados para comparação dos métodos são usualmente empregados na descrição da estrutura de comunidades: número de espécies, porcentagem de recobrimento, índice de diversidade e de uniformidade.

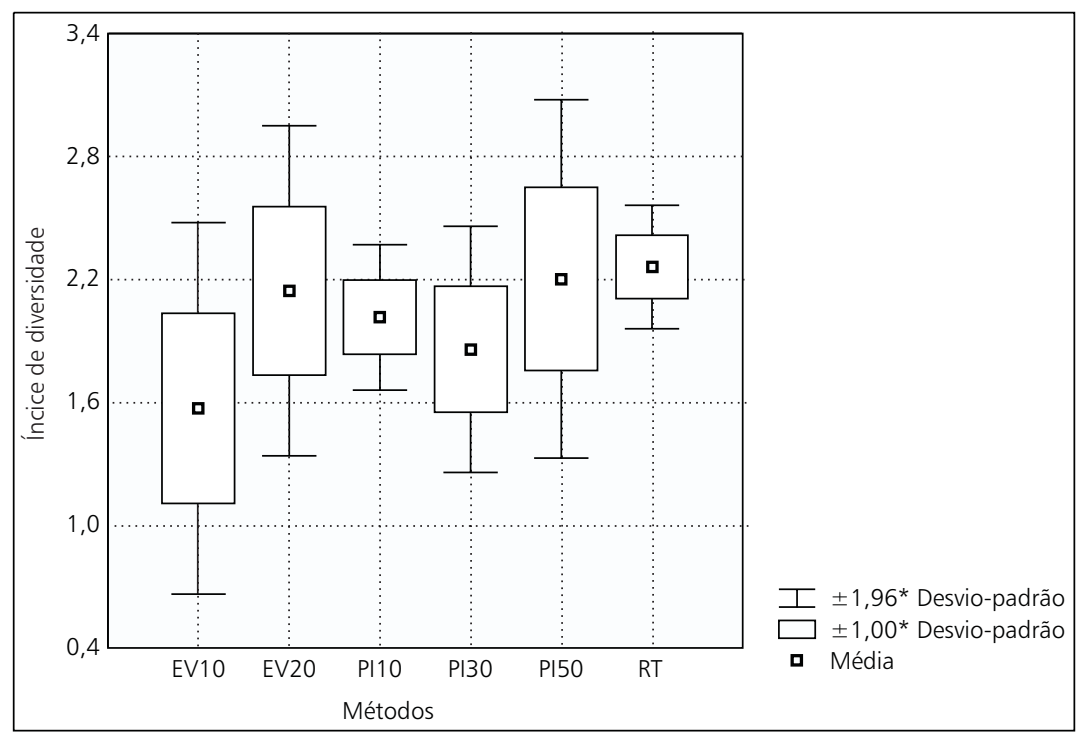

Fig. 6 - Médias dos índices de diversidade obtidas nos seis métodos (siglas conforme explicitado na Fig. 3). 


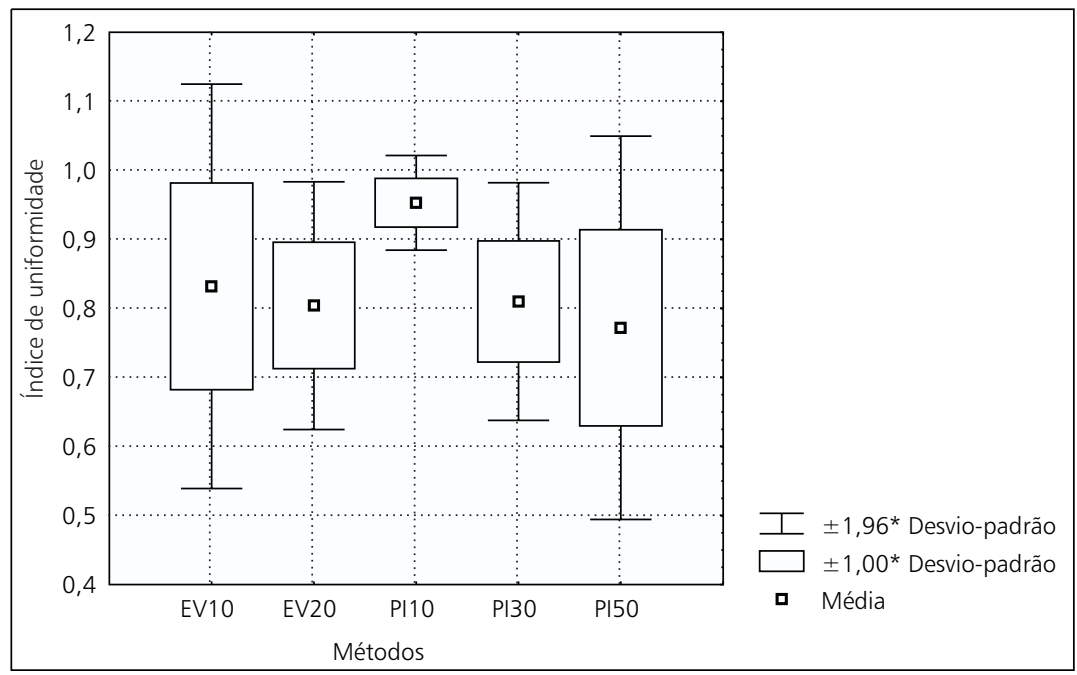

Fig. 7 - Médias dos índices de uniformidade obtidas em cada método (siglas conforme explicitado na Fig. 2).

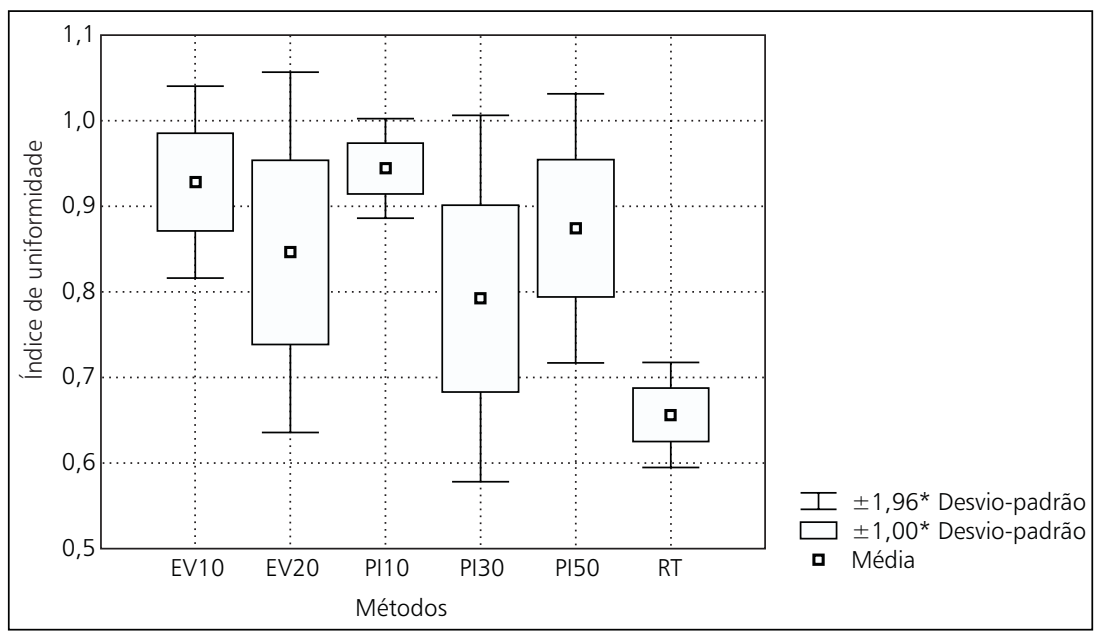

Fig. 8 - Médias dos índices de uniformidade obtidas nos seis métodos (siglas conforme explicitado na Fig. 3).

Em relação às variáveis número de espécies, índice de diversidade e índice de uniformidade, o resultado dos testes indicou, em alguns casos, que há diferença significativa entre os métodos. No entanto, em relação à porcentagem total de recobrimento, a comparação entre os métodos de estimativa visual e os pontos de interseção não apresentou diferenças significativas.

Meese \& Tomich (1992) compararam métodos de amostragem para estimativa da porcentagem de recobrimento abordando também o aspecto da repetibilidade e acurácia dos métodos utilizados.
O resultado mostrou que nenhum método foi significativamente melhor do que outro, resultado que corrobora o que foi encontrado no presente estudo. No entanto, eles encontraram alta variabilidade quando compararam resultados obtidos pelo método de estimativa visual por observadores diferentes na estimativa de uma mesma amostra. Os autores pontuaram essa como uma das principais desvantagens associadas a esse método. Isso indica que o método pode não refletir precisamente o valor real da variável que está sendo estimada, embora Andrew \& Mapstone (1987) afirmem que 
o valor verdadeiro da porcentagem de recobrimento para cada área estudada é difícil de ser conhecido.

$\mathrm{O}$ método de estimativa visual tem como maior vantagem a rapidez na obtenção da estimativa de recobrimento, pois o tempo é um fator crítico quando se estuda comunidades da zona entremarés (Jones et al., 1980; Meese \& Tomich, 1992; Dethier et al., 1993). Dispõe-se de apenas algumas horas durante o período de baixamar para fazer as amostragens.

Um fator a ser considerado nos métodos de estimativa visual é o tamanho dos subquadrados. É preferível maior número de pequenos subquadrados pois ajudam o censo visual quando as espécies encontram-se espalhadas ou apresentam baixo recobrimento. $\mathrm{O}$ uso de grandes subquadrados não fornece ao olho uma referência precisa o suficiente dos contornos dessas subamostras (Dethier et al., 1993).

Da mesma forma, tais métodos não podem se apoiar na criação de complexas regras de decisão, pois diminuem a eficiência amostral e aumentam a variabilidade entre os observadores, o que pode tornar os resultados obtidos por tais métodos incomparáveis.

A comparação em relação ao número de espécies evidenciou que o método que abrange maior área e maior número de pontos de interseção (PI50) registra maior número de espécies, sendo considerado, portanto, diferente dos demais. O restante dos métodos foi agrupado em dois grupos distintos: os métodos de menor dimensão e menor número de pontos (EV10 e PI10) e os de características intermediárias (EV20 e PI30). Os primeiros indicaram menor número de espécies, enquanto o segundo grupo apresentou resultados mais próximos ao de PI50. Resultado bastante coerente com a variável considerada. Menor área e menor número de pontos de interseção registram menor número de espécies e viceversa (Jones, 1980; Pringle,1984; Dethier et al., 1993).

A questão do tamanho ideal da área amostrada é muito antiga. Há alguns anos atrás, junto com a preocupação de conseguir uma representação fiel da comunidade estudada, grande parte dos pesquisadores começou a preocupar se com o tempo necessário para amostragem quando avaliam as dimensões dos seus amostradores (Jones et al., 1980; Pringle, 1984; Meese \& Tomich, 1992). Procura-se atingir o melhor resultado com uma combinação de precisão e rapidez na amostragem, principalmente, nos trabalhos desenvolvidos na zona entremarés. Certamente, a natureza de cada estudo deverá ser considerada na escolha de uma simplificação da metodologia, de forma a não prejudicar os objetivos e o teste de hipóteses do estudo em questão.

$\mathrm{Na}$ questão sobre a área dos amostradores é bastante evidente que a estrutura da comunidade e a faixa de tamanho dos organismos estudados terão um efeito a ser considerado. Na verdade, o objetivo de especificar um tamanho de área amostrada que inclua todas as espécies é praticamente irreal, mas o conhecimento adquirido através das relações espécie/área permite que o efeito dos vários tamanhos de área possa ser avaliado para uma região de estudo (Hawkins \& Hartnoll, 1980). Ballesteros (1986) ressalta, ainda, que o cálculo da área mínima de amostragem de uma comunidade, a partir de poucas amostras, em um única estação do ano é inútil, uma vez que a variação na escala de tempo da riqueza específica e da estrutura da comunidade são suficientemente importantes para que seja necessário um estudo durante o ciclo de um ano. Segundo esse autor, a área mínima deve ser maior do que aquelas encontradas para as diferentes épocas do ano.

Esse mesmo conceito é defendido por Cerqueira et al.(1995) em relação ao índice de diversidade, pois, segundo os autores, o cálculo deste índice pode ser influenciado pelas escalas de tempo e espaço das amostras. A variação na disponibilidade de recursos, bem como flutuações no tamanho das populações e na riqueza de espécies aliado à fragmentação e isolamento dos hábitats estariam influenciando o cálculo da diversidade. Dessa forma, os autores propõem que estudos de diversidade sejam feitos em grandes escalas de espaço e tempo.

Em relação à comparação dos métodos através dos índices de diversidade e uniformidade, os resultados obtidos no presente estudo foram bastante semelhantes. Em cada um dos casos, os métodos apontados como significativamente diferentes tinham características desfavoráveis em relação à variável analisada. Nesse caso, para o índice de diversidade é importante a dimensão do amostrador enquanto que para o índice de uniformidade importa mais a representatividade do método, ou seja, amostrar a maior área possível dentro do amostrador. 
Em relação ao índice de diversidade, o método destacado como diferente dos demais foi EV10, apresentando o menor valor médio para este índice $\left(\mathrm{H}^{\prime}=1,32\right)$. O índice de diversidade considera a relação número de espécies presentes na amostra e a abundância relativa de cada espécie. Uma vez que os valores de índice de diversidade dependem da área amostrada (Ballesteros, 1986), este resultado está relacionado à pequena área desse amostrador $\left(100 \mathrm{~cm}^{2}\right)$ em relação aos demais (400 $\mathrm{cm}^{2}$ ). Essa observação pode ser corroborada pelo fato de que o próximo método na escala de médias foi PI10 ( $\mathrm{H}^{\prime}=1,79$ bits/ind.), que embora amostrasse apenas 10 pontos de interseção, a área amostrada era quatro vezes maior que a de EV10. Segundo Margalef (1980), em alguns ecossistemas a diversidade aumenta quase indefinidamente quando se aumenta o tamanho da amostra. Isso significa que novas espécies são registradas quando se aumenta a área e a representação de suas proporções é flutuante, sendo esse o caso de sistemas mais estruturados. Em outros ecossistemas, a diversidade cresce rapidamente quando se aumenta a área, mas estabiliza-se rapidamente também, sendo esse o caso de sistemas pouco estruturados e, portanto, muito uniformes.

De fato, como discutido anteriormente, os resultados do presente estudo indicaram que o quadrado de $100 \mathrm{~cm}^{2}$ de área (EV10) não foi suficiente para estimar a comunidade em sua estrutura básica, diferenciando esse método dos demais devido ao menor valor médio de índice de diversidade encontrado.

No entanto, quando a comparação foi feita através do índice de uniformidade, uma medida relativa da diversidade, o método considerado diferente dos demais foi PI10. Esse resultado indica que, de acordo com a área do quadrado, poucos pontos de interseção marcados podem acarretar uma amostragem tendenciosa. Dethier et al. (1993) e Foster et al. (1991) afirmam que a precisão dos métodos de pontos de interseção aumenta com o número de pontos marcados ou com o aumento do número de quadrados amostrados.

O método de raspagem total apresentou o menor valor de índice de uniformidade, embora tenha registrado o maior valor de número de espécies e de índice de diversidade. Atribuiu-se essa diferença à própria manipulação da amostra que favorece sua desagregação, podendo conduzir a valores tendenciosos de estimativa de recobrimento para as espécies encontradas, potencializando as diferenças entre as dominantes. Isto, particularmente, aplica-se ao presente estudo, uma vez que as algas amostradas formavam densos tufos, muito compactos, que se desagregavam no momento da raspagem. Dessa forma, a estrutura básica da comunidade encontrada pode não corresponder à observada em campo ou à registrada pelos métodos não-destrutivos.

Para o cálculo do índice de uniformidade, parece ser mais interessante utilizar métodos que considerem toda a área do amostrador (estimativa visual) ou que, dentro de uma área, grande número de pontos de interseção (em relação a área do amostrador) seja amostrado; uma vez que esse índice está relacionado ao grau de recobrimento de cada espécie em relação às demais. Em comunidades em que existe dominância isolada de poucas espécies, a chance de amostrar somente as espécies dominantes aumenta com a diminuição da quantidade dos pontos marcados em relação à área do amostrador, talvez propiciando a caracterização de uma falsa homogeneidade.

Meese \& Tomich (1992) não consideraram a variação no número de pontos de interseção em seus amostradores, mas apontaram esse método como um dos que resultaram em maior variação entre os observadores e as amostragens, principalmente, quando comparado ao método de fotografia com posterior digitalização para análise em computador.

Dethier et al. (1993) observaram que aumentando o número de pontos aleatórios de 50 para 100 havia uma melhoria na precisão e uma diminuição na variabilidade das estimativas. No presente estudo, no entanto, não foi observada diminuição dessa variabilidade com o aumento do número de pontos.

Meese \& Tomich (1992) consideram que existem algumas desvantagens associadas ao método de pontos de interseção, como o tempo necessário para a amostragem e a subjetividade associada à identificação do organismo que está sob o ponto marcado. Em relação ao tempo de amostragem, os autores defendem a idéia de que quando utiliza-se métodos mais rápidos, o aumento no número de amostras pode compensar favoravelmente o pequeno aumento na precisão quando utiliza-se qualquer dos métodos de pontos de interseção. Essa opinião também é compartilhada por Dethier et al. (1993) e Andrew \& Mapstone 
(1987) quando afirmam que deve ser avaliado o ganho real do estudo quando aumenta-se o número de pontos de interseção e diminui-se o número de amostras. Meese \& Tomich (1992) sugeriram que o benefício sobre os métodos visuais são pequenos e, provavelmente, não justificam a perda de informações devido à diminuição no número de amostras.

Dethier et al. (1993) destacam, ainda, entre as desvantagens do método de pontos de interseção, a tendência de não registrar as espécies raras e a limitada precisão da amostragem com uma única amostra (dependente do número de pontos usados).

O método de raspagem total é bem distinto dos demais. Para a variável número de espécies, o resultado foi o esperado, uma vez que a simples observação dos dados evidenciou que através de raspagem total registra-se um número maior de espécies. Esse fato está relacionado à própria metodologia de amostragem, que implica posterior análise do material coletado, o que permite uma triagem cuidadosa da amostra. Uma vantagem associada a este método foi a reduzida variabilidade dos valores dos índices de diversidade e uniformidade quando comparados aos demais métodos.

$\mathrm{O}$ índice de diversidade parece não ser um bom parâmetro para testar diferenças entre métodos, uma vez que os resultados mostram que todos os métodos que utilizam amostradores de mesmas dimensões comportam-se de forma semelhante. Na comparação entre todos os métodos, incluindo-se o de raspagem total, somente EV10 foi considerado diferente dos demais, ou seja, o único de tamanho diferente dos demais.

O método de raspagem é o mais indicado para levantamentos florísticos e estudos fitosociológicos (Yoneshigue, 1985; Yoneshigue \& Valentin, 1988; Villaça, 1988), quando é importante utilizar os parâmetros bionômicos para caracterizar a estrutura da comunidade estudada. Na verdade, essa técnica, também conhecida como relevé, é uma das mais completas para os estudos de vegetação (Boudouresque, 1971), no entanto, é uma técnica trabalhosa e demorada no fornecimento de resultados. Atualmente, a preocupação em determinar as mudanças na estrutura de comunidades de costão rochoso, devido aos impactos de ações antrópicas no meio ambiente marinho, requer técnicas mais ágeis de amostragem, mas que, ainda assim, guardem sua eficiência em retratar as caracte- rísticas da comunidade estudada. Sendo assim, os métodos não-destrutivos que utilizam o levantamento dos dados em campo começaram a ser mais utilizados. Estudos têm mostrado que as mudanças impostas às comunidades bióticas podem ser identificadas através de simplificações nas abordagens metodológicas clássicas, adaptando-as aos objetivos do estudo proposto (Pringle, 1984; Kingston \& Riddle, 1989).

Embora no presente estudo tenham sido detectadas diferenças significativas entre os métodos de estimativa visual e os de pontos de interseção, essas diferenças devem-se mais às variações das subamostras de cada método. Com amostradores de mesmo tamanho e um grande número de pontos de interseção não há diferença importante. Ambos os métodos têm vantagens e desvantagens que podem ser compensadas de uma forma ou de outra, e a escolha deve adequar-se ao trabalho a ser desenvolvido e aos objetivos a serem atingidos.

\section{CONCLUSÕES}

No presente estudo foram comparados três dos métodos mais tradicionais de amostragem (estimativa de porcentagem) de recobrimento de comunidades de costão rochoso: estimativa visual, pontos de interseção e raspagem total. Os métodos com metodologia de amostragem semelhante são estatisticamente similares. A diferença existe quando comparam-se os métodos destrutivos aos nãodestrutivos.

$\mathrm{O}$ método de maior número de pontos de interseção e o de raspagem total são os que melhor registram o número de espécies presentes na área amostrada. Os métodos não-destrutivos para a estimativa total de recobrimento não apresentam diferença significativa nos resultados obtidos.

A comparação através do índice de diversidade também não resulta em diferenças significativas, à exceção do método de menor área de amostragem, fator importante no cálculo desse índice.

Para o índice de uniformidade, o método com menor número de pontos de interseção e o de raspagem total apresentam resultados que impossibilitam sua utilização irrestrita. O primeiro, pelo pouco número de pontos marcados em relação à área do amostrador. $\mathrm{O}$ segundo, pela desagregação da amostra, ambos propiciando uma amostragem tendenciosa, talvez alterando a importância relativa de cada espécie. 
Cada método tem suas particularidades, mas todos podem atender aos objetivos de um estudo específico, bastando para tal um conhecimento prévio da comunidade e das limitações inerentes a cada método. Com essas duas informações podese escolher o método mais adequado, porém uma amostragem preliminar será sempre necessária para definir o planejamento amostral que melhor poderá representar a comunidade que se quer estudar.

Agradecimentos - À CAPES, pelo incentivo e fomento às atividades de pesquisa, características que tornaram possíveis o desenvolvimento deste estudo e a capacitação científica de mais um profissional.

\section{REFERÊNCIAS BIBLIOGRÁFICAS}

ANDREW, N. L. \& MAPSTONE, B. D., 1987, Sampling and description of spatial pattern in marine ecology. Oceanogr. Mar. Biol. Am. Res., 25: 39-90.

BALLESTEROS, E., 1986, Métodos de análisis estructural en comunidades naturales, en particular del fitobentos. Oecol. Aquat., 8: 117-131.

BOUDOURESQUE, C. F., 1971, Méthodes d'étude qualitative et quantitative du benthos (en particulier du phytobenthos). Tethys, 3(1): 79-104.

BOUDOURESQUE, C. F., 1974, Aire minima et peuplements algaux marins. Bul. Soc. Phycol., France, 19: 141-157.

CERQUEIRA, R., GENTILE, R. \& GUAPYASSÚ, S. M. S., 1995, Escalas, amostras, populações e a variação da diversidade. Oecol. Brasiliensis, I: 131-142

DETHIER, M. N., GRAHAM, E. S., COHEN, S. \& TEAR, L. M., 1993, Visual versus random-point percent cover estimations: 'objective' is not always better. Mar. Ecol. Prog. Ser., 96: 93-100.

FOSTER, M. S., HARROLD, C. \& HARDIN, D. D., 1991, Point versus photo quadrat estimates of the cover of sessile marine organisms. J. Exp. Mar. Biol. Ecol., 146: 193-203.

GREIG-SMITH, P., 1983, Quantitative plant ecology. 3a ed., Studies in Ecology, 9. Blackwell Scientific Publ. Great Britain, Oxford, 359p.

HAWKINS, S. J. \& HARTNOLL, R. G.,1980, A study of the small-scale relationship between species number and area on a rocky shore. Estuarine Coastal Mar. Sci., 10: 201214

JOHN, D. M., LIEBERMAN, D. \& LIEBERMAN, M., 1977, A quantitative study of the structure and dynamics of benthic subtidal algal vegetation in Ghana (tropical west Africa). J. Ecol., 65: 497-521.
JONES, W. E., 1980, Field teaching methods in shore ecology. In: J. H. Price, D. E. G. Irvine \& W. F. Farnham (eds.), The Shore Environment, vol. 1: "Methods". Academic Press, London and New York, pp. 19-43.

JONES, W. E., BENNELL, S., BEVERIDGE, C., MCCONNELL, B., MACK-SMITH, S., MITCHELL, J. \& FLETCHER, A., 1980, Methods of data collection and processing in rocky intertidal monitoring. In: J. H. Price, D. E. G. Irvine \& W. F. Farnham (eds.), The Shore Environment, vol. 1: "Methods". Academic Press, London and New York, pp. 137-170.

KINGSTON, P. F. \& RIDDLE, M. J., 1989, Cost effectiveness of benthic faunal monitoring. Mar. Pol. Bull. 20(10): 490-496.

LEGENDRE, L. \& LEGENDRE, P., 1983, Numerical Ecology. Developments in Environmental Modelling, 3. Elsevier Scientific Publishing Co. Amsterdam, The Netherlands, 417p.

MARGALEF, R., 1980, Ecologia. Ed. Omega, Barcelona, $951 \mathrm{p}$.

MEESE, R. J. \& TOMICH, P. A., 1992, Dots on the rocks: a comparison of percent cover estimation methods. J. Exp. Mar. Biol. Ecol., 165(1): 59-73.

MURRAY, S. N. \& LITTLER, M. M., 1978, Patterns of algal succession in a pertubated marine intertidal community. J. Phycol., 14: 506-512.

PRINGLE, J. D., 1984, Efficiency estimates for various quadrat sizes used in benthic sampling. J. Fish. Aquat. Sci., 41: 1485-1489.

ROSSO, S., 1995, Dimensionamento amostral em estudos descritivos de comunidade de organismos bênticos sésseis e semi-sésseis. Oecol. Brasiliensis, I: 193-223.

STATSOFT, Inc., 1993, Statistica for Windows (Computer Program Manual). Tulsa, OK: Statsoft, Inc., 2300 East $14^{\text {th }}$ Street, Tulsa, OK 74104.

VILLAÇA, R. C., 1988, Le Phytobentos Infralitoral des Biotopes Sciaphiles dans la Région d'Upwelling de Cabo Frio (Brésil). Tese de Doutorado. Fac. Sci. Luminy. Univ. Aix-Marseille II, 217p.

YONESHIGUE, Y., 1985, Taxonomie et Ecologie des Algues Marines dans la Région de Cabo Frio (Rio de Janeiro, Brésil). Tese de Doutorado. Fac. Sci. Luminy. Univ. AixMarseille II, 466p.

YONESHIGUE, Y. \& VALENTIN, J., 1988, Comunidades algais fotófilas de infralitoral de Cabo Frio, Rio de Janeiro, Brasil. Gayana Bot., 45(1-4): 61-75. 